\title{
Multi-Spectral Imaging for Fruits and Vegetables
}

\author{
Shilpa Gaikwad* \\ Electronics and Telecommunication Engineering \\ Symbiosis Institute of Technology \\ Symbiosis International University \\ Pune, India, 412115
}

\author{
Sonali Tidke \\ Computer Science and Engineering \\ Symbiosis Institute of Technology \\ Symbiosis International University \\ Pune, India, 412115
}

\begin{abstract}
In the field of agriculture, fruit grading and vegetable classification is an important and challenging task. The current fruit and vegetable classifications are done manually, which results in inconsistent performance. There is an influence of external surroundings on this manual classification. Sometimes getting an expert fruit or vegetable grader, is challenging and the performance of that person may become stagnant over some time. With the recent development in computer technology and multispectral camera system, it is possible to achieve an efficient fruit grading or vegetable classification system. In this manuscript, we summarize different automated fruit grading as well as vegetable classification systems, which are based on multi-spectral imaging. We have focused our analysis on four major areas such as varietal identification, fruit quality assessment, classification, and disease detection. From our analysis, we have found that the Partial Least Square Discriminant Analysis (PLS-DA) was most effective for identifying varieties of tomato seeds. For analyzing the quality of pomegranate fruits, the multiple linear regression model was the most optimal method. Multi-Layer Perceptron (MLP) classifier was the recommended method for classifying medicinal plant leaves. A Linear Discriminant Analysis (LDA) based classifier could accurately detect diseases in fruits and vegetables.
\end{abstract}

Keywords-Multi-spectral imaging; fruit grading; vegetable classification; fruit quality; disease detection; fruit maturity

\section{INTRODUCTION}

A multi-spectral image is the set of images for different wavelengths, which is captured throughout the electromagnetic spectrum [2]. Wavelength separation for image data is typically achieved with the help of color filters [3]. Multispectral imaging not only includes visible wavelength but sometimes the wavelength outside the visible spectrum (i.e. infrared and ultra-violet). With the recent advancement in manufacturing techniques, Light Emitting Diodes (LED's) with multiple colors such as red, green, blue (RGB) came into the picture and they became very popular [4]. The information that we cannot visualize with the human eye, can be visualized with the help of multi-spectral imaging. In multi-spectral imaging, there are at least two to five spectral bands involved. A multispectral system consists of the following spectral bands: visible spectra are in the range of 0.4 to $0.7 \mathrm{um}$. The range of nearinfrared spectra (NIR) is 0.7 to $1 \mathrm{um}$, the short wave infrared (SWIR) is visible in the range of 1 to $1.7 \mathrm{um}$, the midwave infrared (MWIR) is visible in the range of 3.5 to 5 um and long-wave infrared (LWIR) is visible in the range of 8 to $12 \mathrm{um}$. These are combined into a single system [5]. Multi-spectral imaging can be done with a minimum of 3 and a maximum of 15 spectral bands. The applications of multi-spectral imaging include: detecting or tracking military targets, detecting landmines, detection of a ballistic missile, weather forecasting, space-based imaging, and investigation of paintings and documents [6]. Fig. 1 shows the schematic of multi-spectral imaging. The light consisting of a large range of wavelengths (including visible and non-visible) is passed through a multi-spectral filtered disk to generate a specific wavelength of light. A fruit sample is kept into a specific wavelength and a camera captures the image of the sample. Now the multi-spectral filter disk is rotated to obtain other slices of the multi-spectral image. Finally, the computer is used to process the multi-spectral image with the help of algorithms to obtain the grading of the fruit/vegetable sample. The camera doesn't have any IR filter attached to it so that all the wavelengths of light can be observed. Fig. 2 shows the overview of multi-spectral imaging for various applications in fruits and vegetables.

\section{A. Benefits of Employing Multi-Spectral Imaging for Analysing Fruits and Vegetables}

By using multi-spectral imaging for analyzing fruits and vegetables, a lot of important information about the fruits can be extracted. The primary use of multi-spectral imaging in the context of fruits and vegetable analysis is to determine the quality of the product in a non-invasive manner. Higher quality fruits can be marked for export to foreign countries. A particular country can establish its reputation for exporting high-quality fruits and vegetables. In the domestic markets, high-quality fruits and vegetables can be sold at a premium price hence motivating farmers to cultivate a better quality of crops. Another application of multi-spectral imaging is to determine the sugar content of fruits before they are ripe. Assessing the sugar content can help in achieving higher prices for fruits that have high sugar content. Each species of fruit and vegetable would generate unique multi-spectral images. Hence, such imaging techniques would help in identifying the breed of the crop so that it can be properly graded. By analyzing such images of a small number of crops in a field, the eventual overall yield of fruits and vegetables from that field can also be predicted. Multi-spectral imaging is also used to identify disease-stricken fruits and vegetables in quality control systems. In this manuscript, we evaluate the various applications of multi-spectral imaging and remark on the most optimal methods of analyzing such images for each area of application. Main areas of application of multi-spectral imaging are:

1. Varietal identification

2. Fruit quality analysis

3. Classification of fruits 


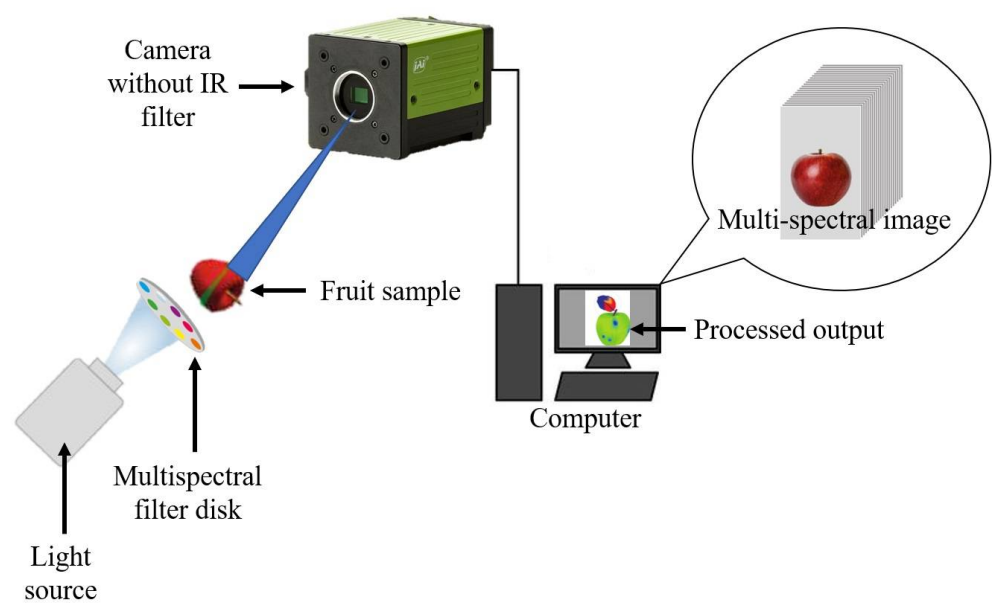

Fig. 1. Schematic Diagram of Multi-Spectral Imaging. The Light Source Wavelengths (Including Visible and Non-Visible) are Passed to a Multi-Spectral Filtered Disk to Generate a Specific Wavelength of Light. A Fruit Sample is Kept at a Specific Wavelength and a Camera Captures the Image of the Sample. Now the Multi-Spectral Filter Disk is Rotated to Obtain Other Slices of the Multi-Spectral Image. Finally, the Computer is used to Process the Multi-Spectral Image with the Help of Algorithms to Obtain the Grading of the Fruit/Vegetable Sample. The Camera used does not have any IR Filter so that All Wavelengths of Light can be Observed.

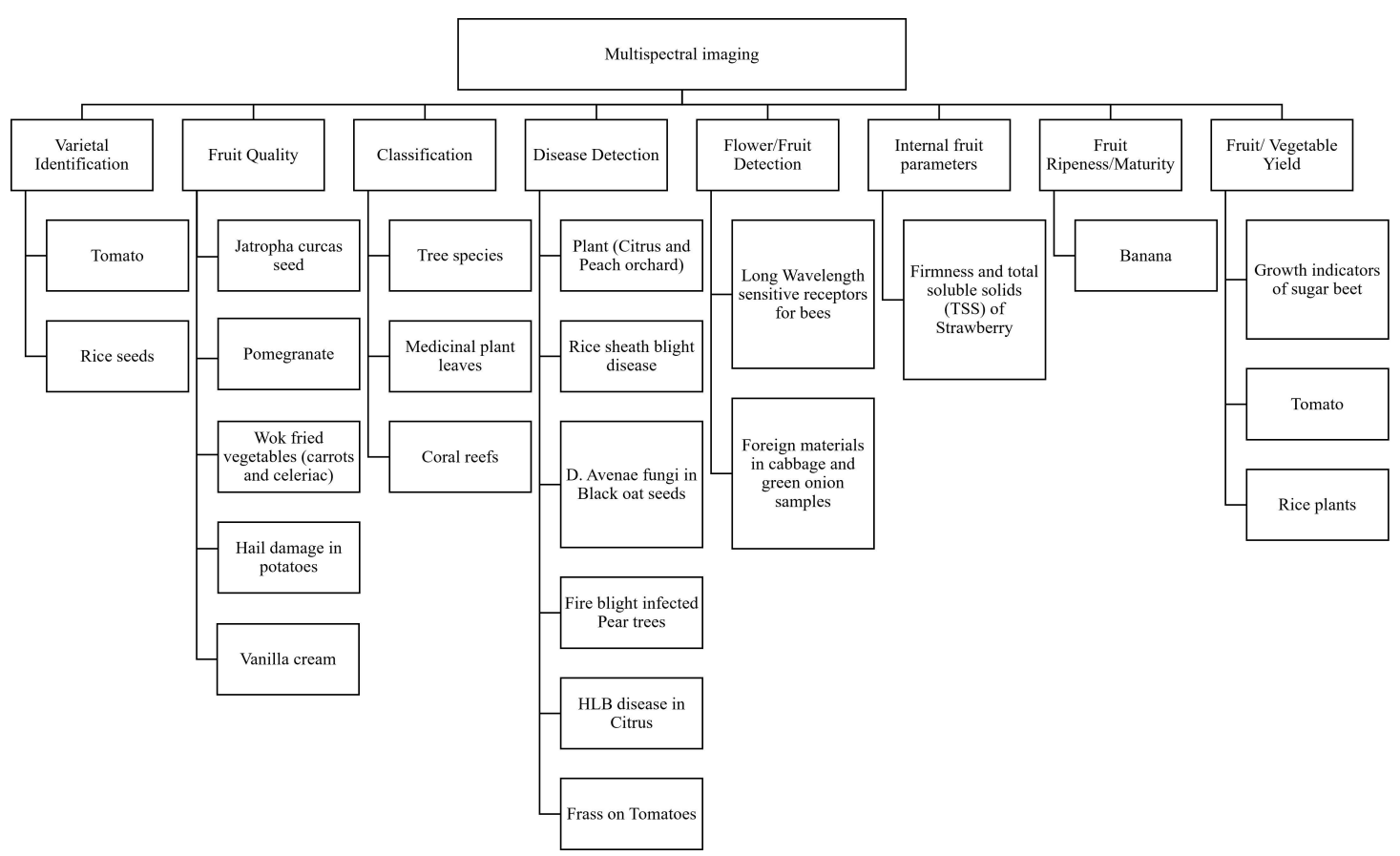

Fig. 2. Overview of Applications of Multi-Spectral Imaging related to the Analysis of Fruits and Vegetables. Varietal Identification is Generally used with Tomato and Rice Seeds. The Quality of Pomegranate Fruits and Jatropha Curcas Seeds can be determined by using Multi-Spectral Imaging. Also, Assessment of Hail Damage Analysis and Differentiation of Vanilla Cream Samples into Fresh and Spoiled Classes can be Performed. It can be used for Classifying Medicinal Plant Leaves, Tree Species and Coral Reefs. Fruits and Flowers can be Identified based on their Multi-Spectral Images. Internal Fruit Parameters are used to Calculate Total Soluble Solids and Firmness in Fruits are measured. The Fruit Ripeness or Maturity Detection can be Performed for Bananas. Another Application of Multi-Spectral Imaging is Fruit and Vegetable Yield Prediction. 

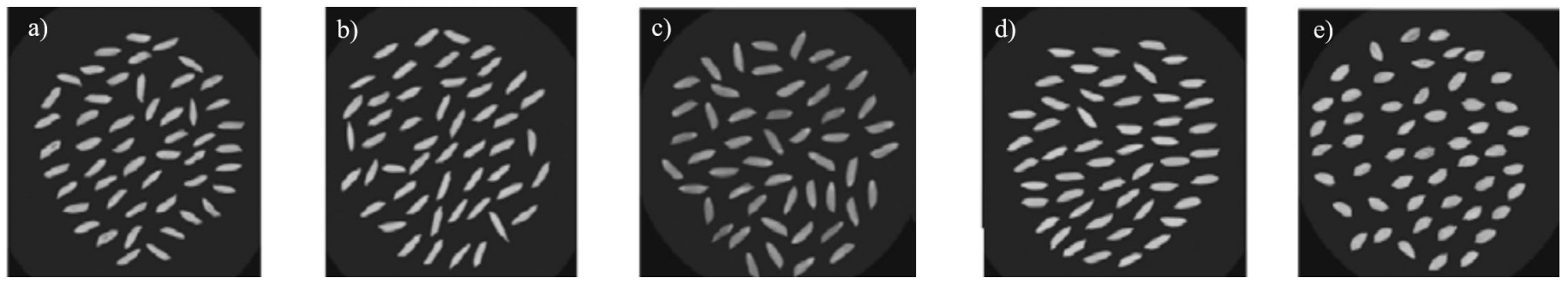

Fig. 3. (a-e) Rice Seeds Classification. Multi-Spectral Imaging Enables the Rice to be Classified as FD2 (a), QXY512 (b), HXD3 (c), QXY822 (d), and WKJ11 (e). These Five Types are Varietal Identification Numbers of Rice [1].

4. Disease detection

5. Flower or fruit detection

6. Internal fruit parameter extraction

7. Fruit ripeness / maturity estimation

8. Fruit Yield

\section{LITERATURE REVIEW}

Fruits and vegetables are very important sources of our daily nutrition. From a consumer's point of view, the quality of fruits and vegetables is a vital feature. Fruit and vegetable quality include both internal and external properties. The internal quality is mainly determined by smell, taste, texture, firmness of flesh, diseases, organic residues, and nutritional quality which includes sugar content, starch, organic acids, total soluble solids content, etc. The external quality is mainly determined by shape, size, color, appearance, and bruises [7].

\section{A. Varietal Identification}

Quality and quantity of yield production are estimated by using a varietal identification-based techniques. Liu et al. [1] used a combination of multi-spectral imaging and chemometric data to find the varieties of rice seeds in a fast manner without damaging the rice seeds. In this study, multi-spectral imaging methods were applied for the separation of rice seeds based on different varieties. Morphological and spectral features were extracted from multi-spectral imaging. Different chemometric methods along with the Partial Least Squares Discriminant Analysis (PLS-DA), Least Squares-Support Vector Machine (LS-SVM) models, and Principal Component Analysis-Back Propagation Neural Network (PCA-BPNN) were used. In the next step, their discrimination performance was compared to classify the rice seeds into five different varieties. The spectral data consisted of various rice seed features. Lastly, the spectral and morphological information were combined respectively and the discrimination performance was compared. After comparing, some differences were found in the varieties of rice seeds and they could be classified according to their variety as shown in (Fig. 3(a-e)). The classification accuracy was up to 94\% for the LS-SVM model, $62 \%$ for the PLS-DA, and $84 \%$ for the PCA-BPNN models.

Recently, Shrestha et al. [8] has proposed a method of varietal separation and recognition of tomato cultivars using Principal Component Analysis (PCA) along with Normalized Canonical Discriminant Analysis (nCDA). They aimed to calculate and compare the parents' and next-generation results obtained by using the method. They considered two sample sets out of which the first set comprised of two cultivars and their two crosses for studying parent and offspring affinity. 11 cultivars were used in the second sample set for varietal identification. With the help of a VideometerLab instrument, multi-spectral images were captured. A blob database was constructed with images of all seeds. Blob feature RegionMSI (mean) could achieve the best separation when it was compared with the intensity of the pixel (mean). The calibration set values are compared with the results obtained from the unknown set. The model could accurately identify the different varieties with an accuracy of $82 \%$. For step-wise PLS-DA models, the classification error was $7 \%$ for the cross-validation dataset as well as the test dataset.

1) Varietal Identification Setup: Images from each seed sample (tomato or rice) are captured using a VideometerLab instrument as shown in Fig. 4. This setup captured multispectral images in 19 different wavelengths such as 375,405, 435, 450, 470, 505, 525, 570, 590, 630, 645, 660, 700, 780, $850,870,890,940$ and $970 \mathrm{~nm}$ [8]. It consists of a sphere that has a white coating to take care of the light getting uniformly scattered around the object. The sphere consists of 19 LEDs along its rim along with a camera that is mounted at the top. Initially, a standard target was used to calibrate the system radiometrically and geometrically. A light setup was employed depending on the type of the sample to make sure that direct comparable images were captured [1]. The background information in the captured image is not relevant hence, a CDA method was used to remove it and only retain the Region of Interest (ROI). The VideometerLab software was used to extract data and transform the pixel data once the images were captured. Various algorithms such as n-CDA, PCA, PLS-DA, BPCNN, and LS-SVM were used. The seed images were segmented based on a certain threshold value. Morphological features such as the area $\left(\mathrm{mm}^{2}\right)$, width/length, and roundness values of seeds were extracted for analysis of the image [1].

\section{B. Fruit Quality}

The quality of fruit is a vital factor when considered from point of view of the consumers. Human experts can examine the quality of the fruit [7]. But manual sorting by visual inspection is work-intensive, slow, inconsistent, and incorrect. With the invention of multi-spectral imaging techniques, automation of the grading process can be performed which will reduce labor costs, and increase the efficiency and accuracy of the sorting process. Fruit quality includes both internal and external properties. The internal quality of fruits includes taste, texture, smell, flavor, flesh firmness, diseases, 


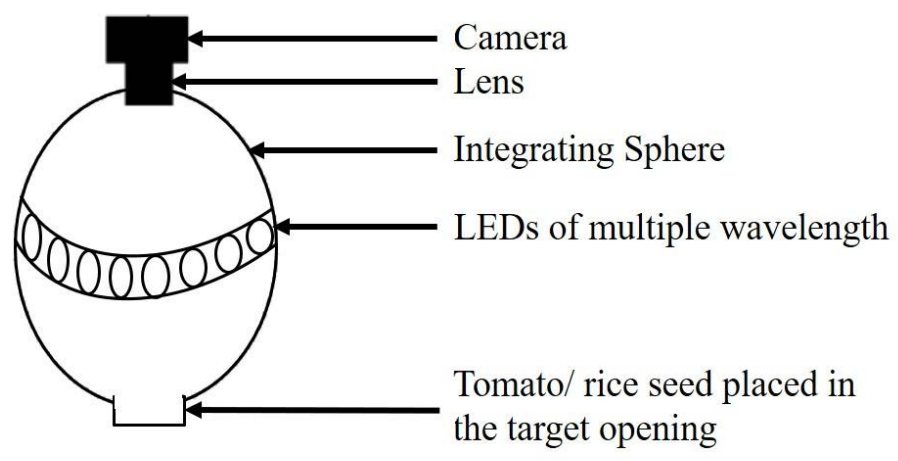

Fig. 4. Structural Setup for Capturing Multispectral Images using the VideometerLab Instrument [8].

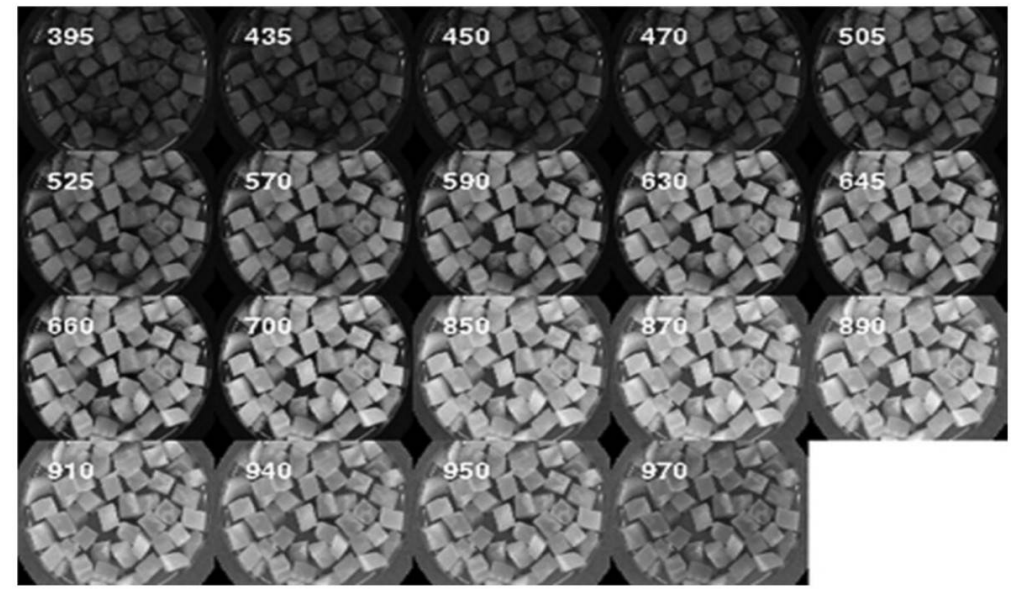

Fig. 5. Plot showing All Spectral Channels of a Celeriac Sample [9].

and chemical/organic residues. It also consists of nutritional quality which includes sugar content, starch, organic acids, total soluble solids content, etc. The quality of fruit depends on the shape, size, color, and skin defects (bruises). The maturity and skin defects are the most crucial features that are used to estimate fruit quality. To evaluate the quality of fruits, internal fruit parameters, ripeness, and yield should be analyzed. An automatic fruit and vegetable grading system will help both farmers and consumers by providing high-quality fruits in the market. Khodabakhshian et al. [10] has developed a multispectral imaging system to assess pomegranate fruit quality. A visible/NIR spectroscopy in the 400-1100 $\mathrm{nm}$ range was used for finding the Total Soluble Solids (TSS), $\mathrm{pH}$, and titratable acidity (TA). The performance of a multi-spectral imaging system was estimated using a multiple linear regression model. The resultant TSS has the value of correlation coefficient (r) as 0.97, the Root Mean Square Error of calibration (RMSEC) was $0.21^{\circ}$ Brix and the Ratio Performance Deviation (RPD) is $6.7{ }^{\circ}$ Brix. Hence, it is shown that the models have a great ability to predict $\mathrm{pH}$ and TA.

1) Block Diagram of Pomegranate Fruit Quality: Spectrometer: As shown in Fig.6 (a) the spectrometer is provided with an optical fibre cable externally. To capture the Vis/NIR diffused reflectance spectra from the pomegranate fruit the measurement system is operated in reflectance mode. For each fruit, an average value of a total of 30 scans was taken. Evaluation of TSS, TA, and pH: Once the spectra were acquired, the TSS, TA, and $\mathrm{pH}$ of each fruit sample were calculated. A manual fruit squeezer was used to find the above-mentioned features. This was followed by the filtration and centrifugation of the fruit. The TSS was measured in ${ }^{\circ}$ Brix three times with the help of a hand-held refractometer. Similarly, a digital $\mathrm{pH}$ meter was used to measure the $\mathrm{pH}$. The average of these measured values for both parameters was recorded. An average percentage of the citric acid was measured after the value of TA was acquired with the help of a Metrohm 862 compact titro sampler. Preprocessing: A huge amount of spectral data is produced with the help of Vis/NIR instruments. However, there is some excessive data captured such as the background noise in the data acquired from the spectrometer apart from the required information on samples. The effect of this irrelevant information is reduced by pre-processing the data. This is done to achieve accurate, stable, and reliable calibration models. The pre-processing techniques utilized for this purpose can be categorized as columns pretreatments and rows pretreatments. ParLeS software was employed to implement the pretreatments. Initially, the average of four spectra was taken to get a single spectrum. An absorbance value was achieved by converting this spectrum using the equation $A b s=\log (1 / R)$ where $\mathrm{R}$ is the quantity 
of reflectance. A linear correlation was achieved between the molecular concentration of the sample and the spectra. Lastly, various methods for pre-processing such as centering, normalization, smoothing, and differentiation were performed. Centering was used to ensure the best results in terms of change across the mean. Smoothing was used to find the best Signal to Noise (SNR) ratio. Multiplicative Scatter Correction (MSC) was used to eliminate the results of scattering related to the average spectrum. Similarly, the background spectra were eliminated and the spectral resolution was increased by using the 1st and 2nd derivative pre-processing methods. Calibration and Validation: A model was developed using the PLS regression method between quality parameters (TA, TSS, and $\mathrm{pH}$ ) from samples and spectral responses of the samples. ParLeS software was used for comparing one quality parameter and the spectral data. The calibration set consisted of 70 samples. The purpose of the PLS method is to find a mathematical relationship between a set of independent variables/predictors which consists of the $\mathrm{X}$ matrix (70 fruits $\times 700$ wavelengths) and the dependent variable which consists of the $\mathrm{Y}$ matrix (70 fruits $\times 1)$. The dependent variables (Y) are defined by the quality parameter (TSS, TA, and $\mathrm{pH}$ ) value from the calibration set. For validation 30 fruits were selected. When the predicted residual error sum of squares was minimum, the calibration model was built using the maximum number of latent elements. The correlation coefficient (r), Root Mean Square Error of Calibration (RMSEC), Root Mean Square Error of Prediction (RMSEP), and Ratio Performance Deviation (RPD) were measured to evaluate the results of the validation and calibration model. Clemmensen et al. [9] proposed an imaging technique for capturing highquality multi-spectral images in carrots and celeriac for 14 days to observe optical reflection changes. For this purpose, the vegetables were fried and frozen at $30^{\circ} \mathrm{C}$ for 4 months before recording a multi-spectral image. Fig. 5 shows the plot of all spectral channels of a celeriac sample. The vegetables were kept at $+5^{0} \mathrm{C}$ throughout the image capturing phase of 14 days. During this period, surface changes, as well as the reflectance properties, were very subtle. But, numerically important differences for some wavelengths and combinations of wavelengths were observed. A t-test was conducted to check for important differences on a $5 \%$ level in many percentage points of the light reflectance. Major variations were observed in the reflectance spectrum of the carrots and celeriac from days 2 to 4 . While for the celeriac, significant changes in the reflectance spectra till day 14 were noted. Bhargava et al. [11] has proposed a computer vision-based method to analyze quality of apples. They have used image processing techniques on the input apple images, along with an Artificial Neural Network (ANN) to achieve $96 \%$ accuracy in quality assessment.

2) Block Diagram of Wok-Fried Vegetables to Find Optical Reflection Changes:: The VideometerLab setup was used to capture the multi-spectral images refer Fig. 6(c). T-tests were performed on the multispectral images to find the importance of reflection changes between days of the experiment. Pixel intensity values of the features were measured at the 5 th, 10th, 25th, 50th, 75th, 90th, and 95th percentiles for each vegetable piece kept in the refrigerator. This was done to examine the reflection changes as a function of days. To categorize the differences 358 carrot samples and 389 celeriac samples were tested in the interval of six different sample days. The pvalues for the T-tests were plotted with the null hypothesis and it showed no variation in mean values for the six-day interval. Lianou et al. [12] proposed an online feature selection grading scheme for vanilla cream quality analysis using multispectral imaging. The study was conducted for the inspection of two microbiological quality classes of cream samples, with the value of total viable counts $(\mathrm{TVC}) \leq 2.0 \mathrm{log} \mathrm{CFU} / \mathrm{g}$ for fresh samples and TVC $\geq 6.0 \log \mathrm{CFU} / \mathrm{g}$ for spoiled samples. They achieved an overall classification accuracy of $91.7 \%$. This model can be further extended to find the microbiological quality of classes by using $1 \mathrm{log}$ step. This step was taken to validate the capability of the model to evaluate increasing microbial populations.

\section{3) Block Diagram to Find Vanilla Cream Quality: Samples} of vanilla cream subjected to microbiological study Fig. 6(c) shows the block diagram of the vanilla cream sample. The vanilla cream sample of $25 \mathrm{gm}$ weight was kept in a sterile stomacher bag having $225 \mathrm{~mL}$ of the sterilized 1/4th concentration solution of Ringer. It was homogenized in a Stomacher device at room temperature for $1 \mathrm{~min}$. To calculate the TVC, precise serial decimal dilutions in Ringer's solution were considered. Finally, colonies were measured after an incubation period of 48 hours with the temperature maintained at $30^{\circ} \mathrm{C}$. The microbiological quality of data was indicated as $\log$ (colony forming units) per gram of cream (log CFU/g). Image capturing and its study: The VideometerLab setup was used to capture the multi-spectral images for each cream sample. Capturing the spectral data, a data cube of $\mathrm{m} \mathrm{x}$ $\mathrm{n}$ size in pixels is captured for each cream sample. This resulted in the production of a huge amount of data describing samples in time-based experiments considering different storage conditions. Before the image was captured a light setup called auto light was used and the calibration was performed radiometrically and geometrically. The samples of vanilla cream were placed in an Ulbricht sphere which has a camera installed on the top and the subsequent multispectral image of the cream surface was captured. To eliminate the redundant data, segmentation was done using the in-house method. After this normalization was done for pixel values of each wavelength in the range $[0,1]$. The next step was to calculate the mean reflectance spectrum and the respective standard deviation values were calculated. Data labelling: Out of the 245 spectra of the cream samples, 65 spectra were used in model training, 48 spectra were used for validating the model and the remaining 132 spectra were used in model testing. The input matrix had 18 average and 18 Standard Deviation (SD) values of the reflective spectra extracted from 245 samples of vanilla cream. The output matrix had the results of microbiological data for finding TVCs in the corresponding samples. At first, binary classification was applied that will automatically find samples having TVC $\geq 6 \mathrm{log} \mathrm{CFU} / \mathrm{g}$ and TVC $\leq 2 \log \mathrm{CFU} / \mathrm{g}$. The model was constructed to correctly find fresh or spoiled samples. Dynamic Feature Selection (DFS) method: An Unsupervised Online Feature Selection (UOS) algorithm based on the DFS method was used to choose the best model features corresponding to certain test data used. A three-step DFS approach was designed. The first step was training-dependent feature elimination. The second step was the online test-dependent feature elimination. The third step was the decision taken by considering the training set and test 
(a)

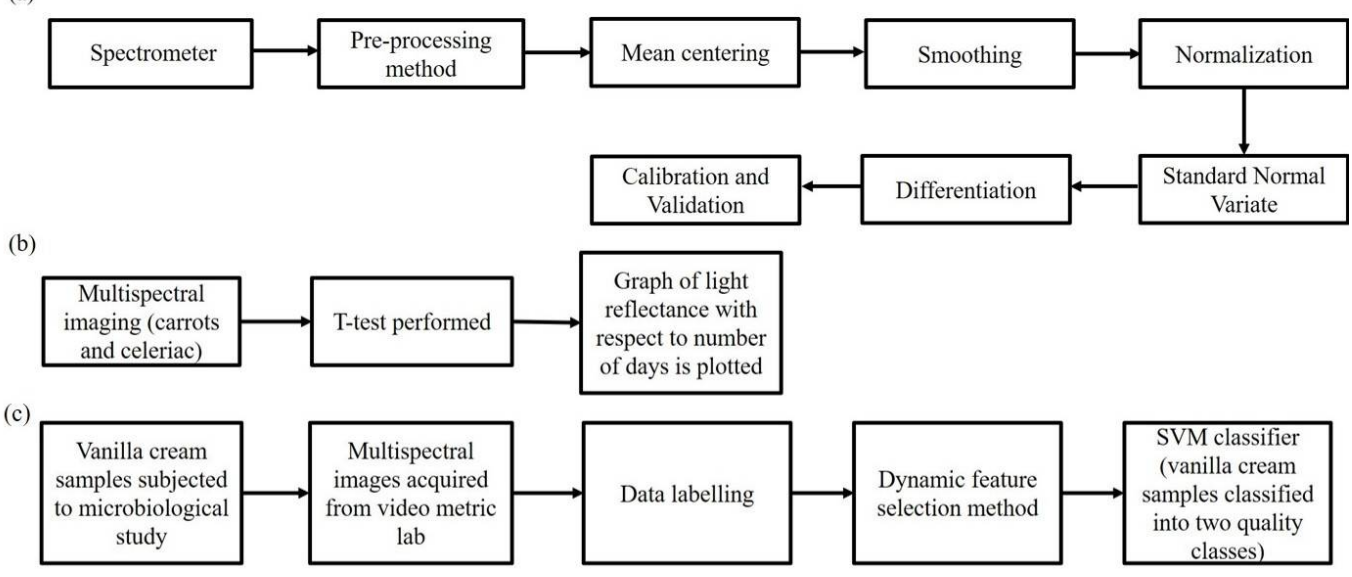

Fig. 6. Block Diagram for Fruit Quality. (a) Chemometric Block Diagram to Extract the Information of Pomegranate Fruit. (b) Block Diagram to Calculate Optical Reflection Changes in Carrots and Celeriac using Multispectral Imaging. (c) Block Diagram of SVM Classifier to Classify Vanilla Cream Samples in Two Quality Classes.

samples collected. The classification model is built when the training set features fit all three conditions for each test sample. Support Vector Machine (SVM) classifier: SVM with linear kernel was used to classify the vanilla cream samples. Every new test sample produces a new set of features. These features are re-inserted in the data set for training and certain features were selected to create new test data in the online feature selection method.

4) Internal Fruit Parameters: Liu et al. [13] proposed the use of multi-spectral Imaging to find quality parameters and ripeness phases of the strawberry fruit. A total of 210 fruits were evaluated. For developing models, the calibration set included 162 fruits which comprised of raw, ripe, and overripe classes with 54 samples each. For model validation, 48 fruits were considered which comprised of raw, ripe, and overripe samples with 16 samples each. This was done so that the calibration model could verify the prediction performance. The results show that for predicting firmness and TSS content, the BPNN model's performance was better as compared with PLS and SVM models using multi-spectral imaging. The SVM and PCA-BPNN models were tested to classify fruit based on the maturity stage with the help of multi-spectral imaging. The SVM model achieved a classification accuracy of $100 \%$.

5) Block Diagram to Find Internal Fruit Parameters (TSS and Firmness) in Strawberry Fruit: Sample preparation and multi-spectral imaging: 210 strawberry fruits were considered (70 raw and 40 ripe samples) (Fig. 13). Multi-spectral images were captured for all the fruits. To generate the overripe fruit category, the ripe fruits were kept at room temperature for two days. A texture analyzer was used to perform penetration tests on the skin of the fruit with a depth of $7 \mathrm{~mm}$. The firmness of the fruit was also calculated. The TSS was measured with a handheld refractometer. The measurement of each fruit was done thrice immediately after the multispectral image measurement. The VideometricLab equipment was used for capturing multi-spectral images of the strawberry fruit. Segmentation of the images into proper regions was performed for image evaluation. The nCDA algorithm was used to remove the background information and segmented using a simple threshold. Otsu adaptive thresholding method was used to emphasize the required features which were followed by segmentation. The images of strawberries after removal of the background were transformed into spectra depending on the calculations of the mean. Each image was contributing its spectra for model calibration. Data evaluation: The model was built by considering the acquired spectra and the quality features like TSS and firmness. The SVM, PLS, and BPNN methods were used to develop the prediction model.

6) Fruit Ripeness or Maturity: With the help of multispectral imaging, a method for estimating the ripeness of bananas is proposed by Santoyo et al. [14]. The brown spots on the banana peel can be separated accurately by employing the Hotelling transform. Two optical filters that were visible in the range of 410-690 nm and NIR in the range of 820-910 nm were used for this purpose. The texture homogeneity criteria were used to specify the growth of the brown spots present in process of ripening with the fusion of spectral image. Fig. 7 shows the 7 phases of the banana ripening process.

7) Block Diagram to Find Banana Ripeness: In this work, seven specimens of bananas were taken. Each day 10 images were taken for every specimen (one for every optical filter) (as shown in Fig. 8). This process resulted in 490 images. The banana specimens were stored for 7 days. 10 different optical filters were used in this experiment. The flat-field correction technique was applied to remove the unwanted signals and retain the useful data for each multi-spectral image. To estimate the maturity of the banana, quantification of the brown spots growing on the peel was carried out. Three quantifying methods namely Fourier fractal analysis, Hotelling transform, and texture analysis were used. These techniques were employed to compute the homogeneity criteria related to the co-occurrence matrix. These methods also helped to find the level of similarity in a set of pixels. The comparison between all three methods was performed to select the one which gave the best results for measuring the ripeness of the banana.

8) Fruit Yield: Cao et al. [15] developed an Unmanned Aerial Vehicle (UAV) to capture multi-spectral images for 
a)
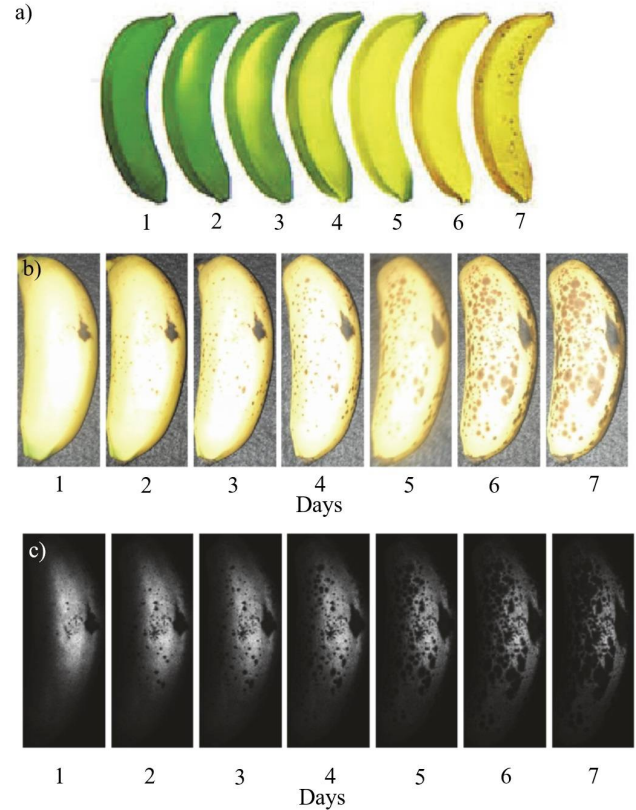

Fig. 7. (a) Seven Phases of the Ripening Process of the Banana. (b) Example of the Images used during the First Week which shows the Colour of the Sample. (c) Example of the Images used during the First Week that shows Gray Scale Images from a Specified Spectral Region for the Same Sample [14].

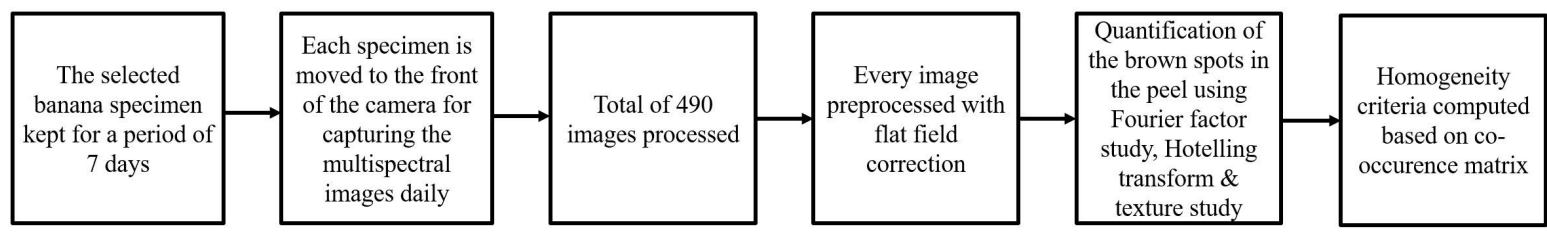

Fig. 8. Block Diagram to Estimate Ripeness of Banana Fruit.

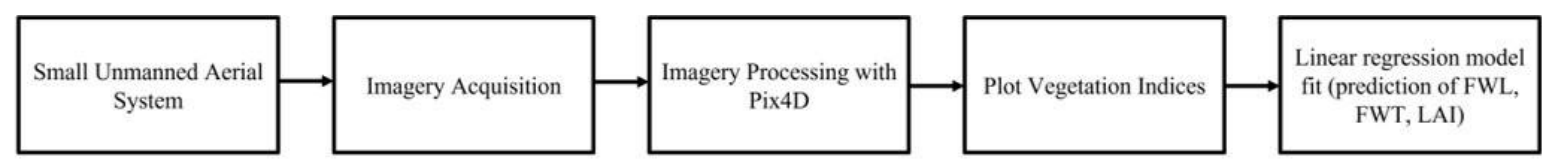

Fig. 9. Block Diagram of Fruit Yield of Sugar Beet Crops.

monitoring growth indicators of sugar beet crops. For this purpose, a wide dynamic range vegetation index (WDRVI) is used. The values achieved for the determination coefficients $\left(\mathrm{R}^{2}\right)$ for leaf area index (LAI), Fresh Weight of Leaves (FWL), and Fresh Weight of Roots (FWR) models of the sugar beet were created using the WDRVI were 0.957, 0.950, and 0.963, respectively. The accuracy of growth indicators of sugar beet can be improved from $1.05 \%$ to $5.07 \%$ using the WDRVI index model. The relation between the ground biomass of sugar beet and the growth indicator helps in finding the accuracy of the growth indicator monitoring. The increase in the ground biomass of sugar beet reduces beet growth indicator, improving the accuracy of growth monitoring by using WDRVI saturation. There is huge biomass in sugar beet and thus, this technique proves to be useful. Wittstruck et al. [16] used UAV-based image data to detect Hokkaido pumpkins and estimate their yield with high accuracy.
9) Block Diagram to Find Sugar Beet Growth: A small UAV was used for aerial remote sensing as shown in Fig. 9. This comprised of MicaSense Red Edge-M multi-spectral sensor and a passive light optical sensor. The images of sugar beet were captured during the crucial growth period which consists of seedlings, leaf plumps growth, root growth, and accumulation period of sugar. The pre-processing of multispectral data was performed to remove noise, distortion in the sensor, removal of background information, and radial correction. 20 points were considered in the test area. In addition to this, a light sensor, GPS, inertial measurement unit was utilized to get the exact spatial data required. WGS84 was used as a geographic coordinate system. By adding the weight coefficient to NDVI, four WDRVI indices were calculated. This was done to evaluate the LAI, FWL, and FWR of the study plots. The sensitivity evaluation was performed based on the five vegetation indices and three growth indicators when NDVI $>0.8$. From the results, it was observed that the WDRVI index is more sensitive than NVDI. It was also seen that the 
correlation coefficient between the beet growth index and the WDRVI index is higher than that of NDVI. The models were developed using the data from the years 2018 and 2019. 70\% of the data was used to develop the model and $25 \%$ was used for verifying the model. RMSE, Relative Root Mean Square Error (RRMSE), $R^{2}$ were calculated. The results show that the WDRVI1 index with a weighted coefficient of 0.05 accurately evaluated the LAI, FWL, and FWR. WDRVI1 evaluated the FWL in the early growth phase in the best way.

\section{Classification}

Naeem et al. [17] applied multi-spectral imaging and texture feature extraction along with a Multi-Layer Perceptron (MLP) classifier to categorize medicinal plant leaves using five spectral bands with a range of $460 \mathrm{~nm}$ to $1560 \mathrm{~nm}$. Six medicinal plant leaves were considered. Fig. 10 shows the grayscale image of the six medicinal plant leaves. The classification accuracy achieved for tulsi was $99.10 \%$, for peppermint $99.80 \%$, for Bael $98.40 \%$, for lemon balm $99.90 \%$ , for catnip $98.40 \%$, and for Stevia $99.20 \%$ using the multilayer perceptron classifier. The MLP method achieved a high accuracy of $99.01 \%$ as compared to the other methods.

1) Block Diagram of Medicinal Plant Leaves Classification: A computer vision-based experimental setup was used for collecting multi-spectral and digital images as shown in Fig. 11(a). The images were transferred into the grey level format with a resolution of $800 \times 800$ pixels by cropping the leaf region exactly. A Sobel filter was used for edge/line detection of seed intensity. If the seed intensity threshold value is greater than 6 , then mark the region as a region of observation. In this work, five regions of observation were drawn on each image and fused features were extracted from the data set. The chisquare feature selection technique reduces the feature vector space and selects the fourteen best features for medicinal plant leaves classification. Five machine learning classifiers namely MLP, LogitBoost (LB), Bagging (B), Random Forest (RF), and Simple Logistics (SL) were employed on the medicinal plant leaves database. Abdollahnejad et al. [18] proposed a method for tree species classification and health condition assessment with the help of Unmanned Aircraft System (UAS) multi-spectral imaging for the mixed broad leaf-Conifer forest. They developed a method to recognize healthy, unhealthy, and dead trees affected by bark beetle infection. The results show an overall accuracy $(\mathrm{OA})=81.18 \%$ and values of Kappa $=$ 0.70 which prove the capability of their method to classify tree species. The health status condition of tree species shows the value of an $\mathrm{OA}=84.71 \%$ and $\mathrm{Kappa}=0.66$. The SVM method proved as a good classifier of tree species. The fusion of vegetation indices (VI) and texture analysis (TA) layers also results in increased OA by $4.24 \%$. Their method can be used to check huge areas affected by biological intrusion factors for mapping and detection very fast. Other applications of their proposed method are to estimate habitat conditions and tree inventory at low costs.

2) Block Diagram of Tree Species Classification and Health Status: UAS bi-temporal aerial imagery was used which incorporates a high-resolution 5-band spectrum as shown in Fig. 11(b). In the pre-processing step, the MicaSense RedEdge-M multi-spectral equipment was used to calibrate the reflectance panel and to convert raw pixel values into reflectance. This was performed to correctly describe the target consisting of the object of interest from the image pixel values. In the absence of calibration, the data collected will not be correctly compared for change identification. Using Structure from Motion (SfM) imaging photogrammetric database was generated. The Agisoft Metashape is used so that the values of image pixels correctly identify the region of interest by adjusting the atmospheric and light conditions For the canopy spectral study and texture study, DTMs, DSMs, and orthophotos were given as input with a resolution of $0.05 \mathrm{~m}$. For the spectral study, $9 \mathrm{VI}$ and 13 TA variables were used. After this bi-temporal data was combined with texture data. Then supervised machine learning technique was employed to catch the reflectance values within a buffer area of $2 \mathrm{~m}$ radius from each treetop. Using the same buffer area effect of crown shape and vague pixels from the overlapped crown areas were removed. The SVM algorithm delivered good results and could achieve the correct and reliable classification of tree species.

3) Fruit or Flower Detection: Vasas et al. [19] developed a technique for edge detection in bees in which multispectral images of flowers show the importance of utilizing long-wavelength sensitive receptors. With the help of multispectral image dataset of flowering plants, SNR ratios of longwavelength $(\mathrm{L})$ receptor reply were four times higher than the short $(\mathrm{S})$ and medium $(\mathrm{M})$ wavelength receptors depending on the specific conditions. The band-pass filters included the full wavelength range of bee vision which had transmission peaks at 340, 400, 460, 520, 580, 640, and $700 \mathrm{~nm}$. As shown in Fig. 17 honeybees and bumblebees collect visual data with the help of 3 types of photoreceptors. However, only a single type of receptor is used that responds to longer wavelengths that are used for movement and edge detection. The results show peak sensitivity at $544 \mathrm{~nm}$ which corresponds to green for longwavelength receptors which give the most steady signals in response to the natural objects.

4) Block Diagram to Detect Long Wavelength Receptor Responses of Bees: The database consisting of 53 images was scanned and processed in the ImageJ software as shown in Fig. 18. Java plugin color correction was used to adjust the white balance. The normalized cross-correlation (NCC) method was used to separate the images of the same flower. Different parts of the flowers such as petals, centers, and leaves were manually selected for comparing the reflectance spectra. A total of 52 images were captured. 1000 pixels were randomly selected from the different parts of the flower using which the standard deviation, mean, and SNR were calculated. To understand the difference between the vision of humans and bees, false-colored images were added to the database. The black and white images were tinted to the hue set by the filter wavelength with the help of ImageJ. The resultant images were combined with the RGB color images. The combination of RGB layers produces an original color in human vision. However, for bees, the UV, Blue (B), and yellow $(Y)$ layers are tinted with $B$, Green $(G)$, and Red $(R)$ to produce false-color images. Calculating Receptor Responses: The Standard deviation (SD) and mean was calculated for the quantum catches $(\mathrm{P})$ and relative receptor responses $(\mathrm{E})$ which are functions of receptor peak sensitivities. Lastly, the effects of reflectance functions, illumination spectrum, background reflectance spectrum, the shape of the sensitivity of the receptor were evaluated. These results were combined 


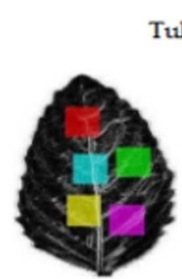

Front

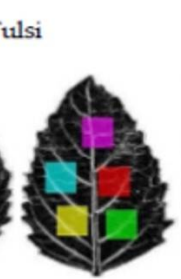

Back

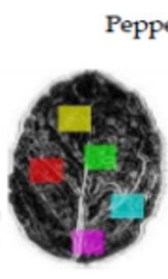

Front

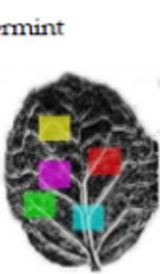

Back

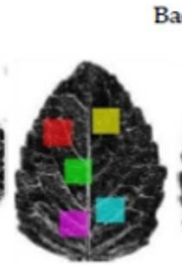

Front
Bael

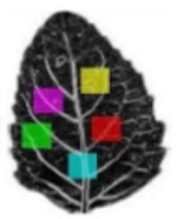

Back

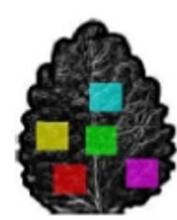

Front

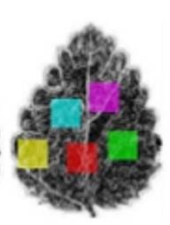

Back

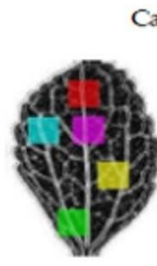

Front
Catnip

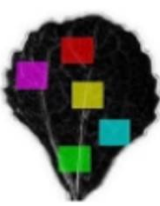

Back

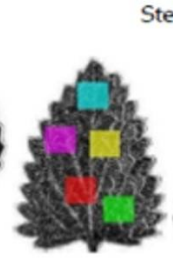

Front

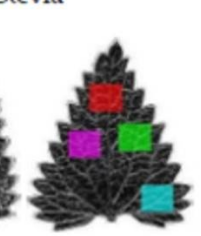

Back

Fig. 10. Images used for Medicinal Plant Leaves Classification. The Original Images are Transformed into Grayscale with the ROI that Allows the Classification of these Leaves into Tulsi, Peppermint, Bael, Lemon Balm, Catnip and Stevia. It also shows Five Colorful Regions of Observation [17].

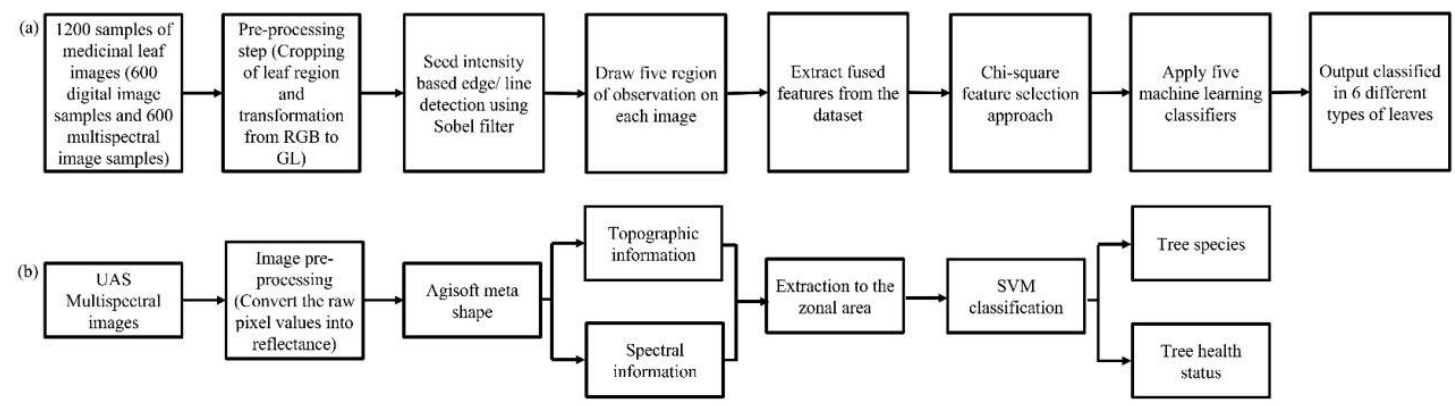

Fig. 11. Different Classification Strategies using Multi-Spectral Imaging on the Plant Data. (a) Block Diagram for Medicinal Plant Leaves Classification. (b) Block Diagram of Disease Detection in Tree Species to Recognize Healthy, Unhealthy and Dead Trees Affected by Bark Beetle Infection.

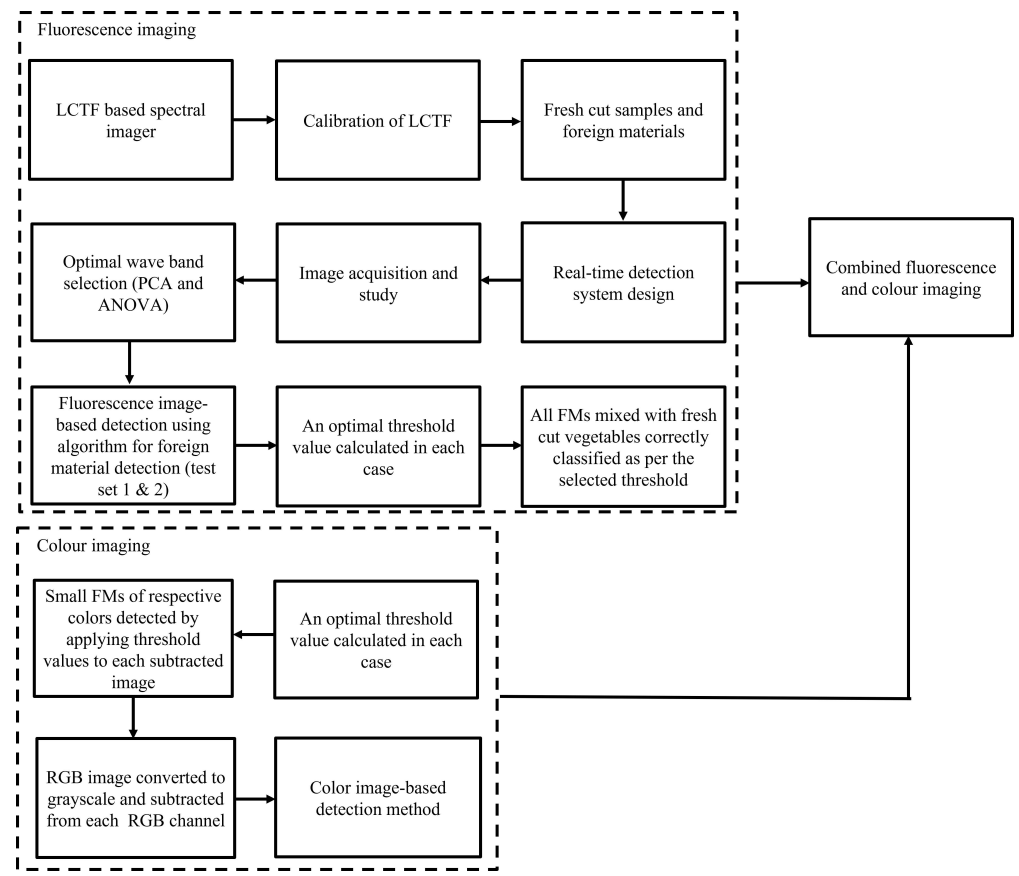

Fig. 12. Block Diagram for Detection of Foreign Material in Cabbage and Green Onion Samples using Reflectance and Fluorescence Images. 


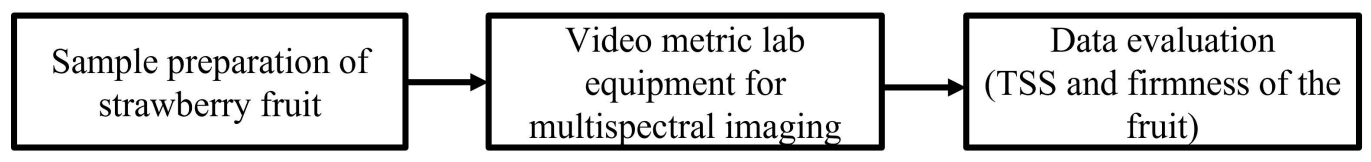

Fig. 13. Block Diagram of Internal Fruit Parameter in Strawberry Fruit (Firmness and TSS).

and given as input to the null model. To get a more clear picture of the bee's vision, heat maps were included which show the receptor responses of the long, medium, and shortwavelength for each multi-spectral image. A real-time system was developed by Lohumi et al. [20] for the identification of foreign materials (FMs) mixed with fresh-cut vegetables, with the help of fluorescence and color imaging. The setup included a multi-spectral fluorescence imager merged with a Liquid Crystal Tunable Filter (LCTF) to capture desired band images sequentially of fresh-cut vegetables. Fig. 13 shows the color images of FM with cabbage sample. The detection accuracy (average) of FMs in cabbage and green onion sample was considered. The average of the total detection accuracy for 4 repetitions was calculated to get the detection accuracy, which outperformed $95 \%$. A processing unit for vegetables that were fresh-cut was installed in an industrial environment to further test the real-time detection system. The performance of the developed real-time system was almost the same in the industrial condition. The system can scan an area of a maximum of $24 \times 24 \mathrm{~cm}^{2}$ in a time duration of approximately $1.5 \mathrm{~s}$.

5) Block Diagram to Identify FM in Fresh Cut Vegetables: As shown in Fig. 12 the LCTF based spectral imager was employed to focus a UV-A beam light onto the target area. A built-in white light was used to provide an alternative for the changeover. Thus, both reflectance and fluorescence images could be captured with this setup. Fresh cut samples and FMs: In this work, cabbage and green onion samples were used in response to the fluorescence and change in color as shown in Fig. 19. The FMs that were added to the freshly cut vegetables are plastic pieces, peel residues, small metal pieces, and various kinds of woods pieces, toothpicks, etc. Real time detection system design: White LED illumination was used to capture the color images of the samples on the conveyor unit. Fluorescence and color imaging units were placed in such a manner that they did not interfere with each other. The movement of the conveyor belt was controlled using a motion controller board and a computer unit and synchronized with the sensing unit. This was done to capture the fluorescence images of the samples placed on the conveyor unit. This was followed by, capturing the color images of the same area when the sample moved one step ahead, which corresponds to approx. $24 \mathrm{~cm}$, of the Field of View (FOV) of the color camera. Classification using optimal waveband selection: The PCA and ANOVA methods were used to reduce the volume of data and select the optimal wavelength. In this study, differentiation of vegetables and FMs on the conveyor belt as well as the differentiation of the vegetable samples from the FMs was required. Thus, the best wavelengths were chosen to visually inspect the fluorescence spectra of the conveyor belt, fresh-cut vegetables, and FMs. The maximum peak intensity centered at a wavelength of $465 \mathrm{~nm}$ was chosen from the fluorescence spectra of the conveyor belt. The fluorescence peak intensity value was either $465 \mathrm{~nm}$ or $615 \mathrm{~nm}$ for different FMs. The fluorescent FMs have been recognized using these 2 band wavelengths. But the $465 \mathrm{~nm}$ waveband created a problem for fluorescent FMs as the images were partly covered by the fluorescent signals of fresh-cut vegetables. To solve this problem, a $435 \mathrm{~nm}$ wavelength image was used where a little variation is observed between the fluorescent intensity of freshcut vegetables and the FMs. Later these two band images were averaged (435 $\mathrm{nm}$ and $465 \mathrm{~nm}$ ) and given a threshold to check the existence of fluorescent FMs. Flourescence image-based detection: Two tests were performed with an algorithm to detect the FMs. A threshold value was set in each case of the two tests by examining the smallest number of resulting false positive and false negative pixels. Based on the threshold, the classification of the FMs mixed with fresh-cut vegetables was done accurately with minimum error. Color imagingbased detection: Initially, the RGB image was transformed into a grayscale image, and the image was subtracted from each RGB channel. The threshold values which were set were applied to each subtracted image to detect the small FMs of corresponding colors. For the smallest number of resulting false positive and negative pixels, the best threshold value was selected in each case. It was observed that the accuracy of the color imaging-based detection method is less than that of fluorescence imaging. Combination of Color and fluorescence imaging: The combination of fluorescence and color imaging gives better accuracy. This technique gives much higher accuracy as compared to that of the single imaging technique. By counting the mutually independent detected FMs from the RGB-based and fluorescence-based classification images, the combined accuracy was calculated. In this work, combined detection accuracy (average) for 4 repetitions of the test exceeded $95 \%$.

\section{Disease Detection}

Zhang et al. [22] used multi-spectral imaging for the detection of rice sheath blight $(\mathrm{ShB})$ disease with the help of an unmanned aerial system. The results indicate that between ground-measured Normalized Difference Vegetation Index (NDVIs) and image-extracted NDVI's there was a strong correlation. The determination coefficient $\left(\mathrm{R}^{2}\right)$ is 0.907 and the RMSE is 0.0854 . Whereas the results indicate that between image extracted NDVIs and disease severity there is a good correlation with a value of $\left(\mathrm{R}^{2}\right)=0.627$ and the RMSE $=0.0852$. NDVIs calculated from the images achieved an accuracy of $63 \%$ which could measure various levels of the disease in the plots. The study included original RGB and hue, lightness, and saturation (HLS) transformation images of 67 field areas.

1) Block Diagram for Rice ShB Disease Detection: Unmanned aerial system (UAS): To capture the imagery data from the field plot, a UAV along with a higher resolution multispectral camera was employed (refer Fig. ??). The Micasense 


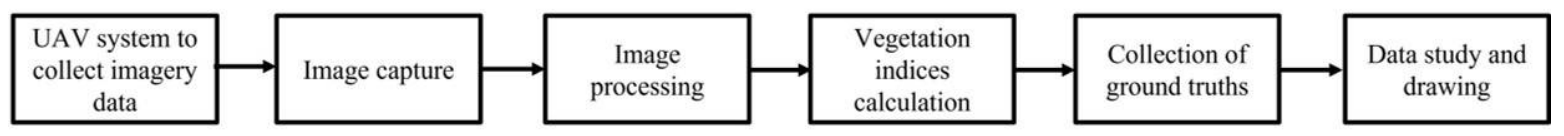

Fig. 14. A Generalized Approach for Rice Sheath Blight (ShB) Disease Detection using Multi-Spectral Imaging to Estimate Severity on a Rated Scale (0 to $\pm 9)$.

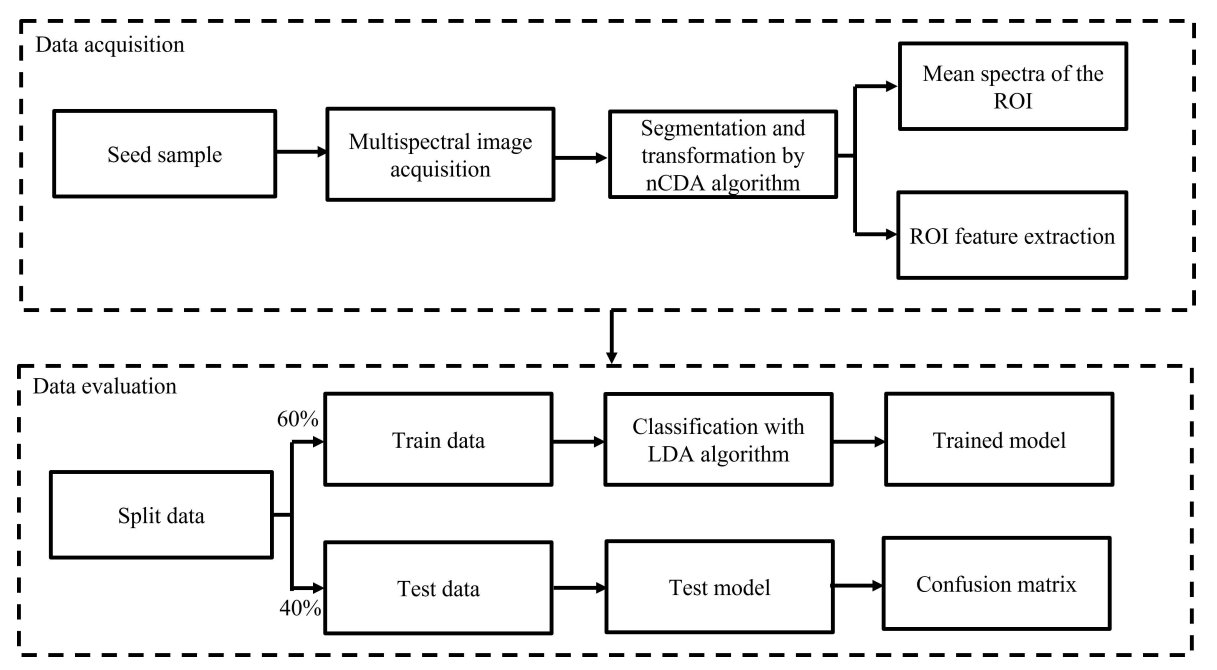

Fig. 15. Block Diagram to Differentiate between Fungi-Infested and Non-Infested Black Oat Seeds using Spectral Information.

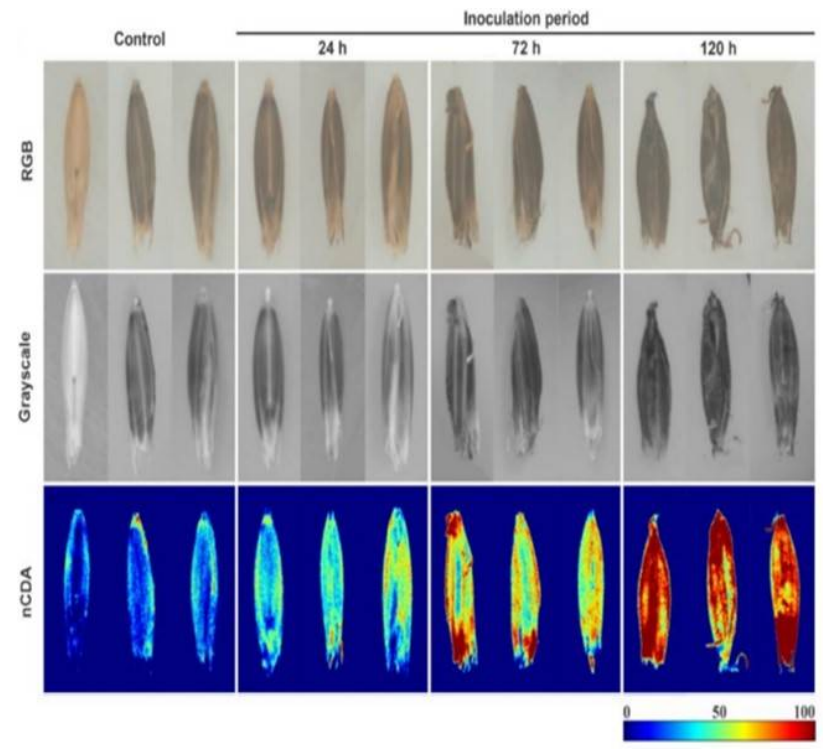

Fig. 16. Images of Black Oat Seeds: Raw Images and Corresponding Grayscale and nCDA at Wavelength 365 nm for Seeds Free from Fungus and Seeds Exposed to D. Avenae Fungi for 24, 72, and 120 Hours. The Blue Color Defines Healthy Tissues, Intermediate Contamination is Defined by Green and Yellow Colors and the Red Color Defines Large Contamination in Fungi in the Images Transformed by the nCDA Algorithm [21]. 
RedEdgeTM can capture 12-bit raw images in 5 narrow bands. This camera was used to calculate the reflectance of the plant which contains data about stress in crops more accurately than the 3 channel camera. Image capture: To cover all 67 field plots the camera was directed at the nadir at two heights, 27 meters above the ground and 5.5. meter to cover 4 plots for every image during the flight. Image pre-processing: To acquire color traits from the captured images and conversion of these images into various color spaces, ENVI software was employed. In this work, digital images of various severity levels of ShB were converted to lightness, hue, and saturation. After this, the mean values of HLS were taken. VI calculation: 5 types of VI's - NDVI, Ration Vegetation Index (RVI), Difference Vegetation Index (DVI), Normalized Difference Water Index (NDWI), and Red Edge (RE) were measured from the captured multi-spectral images. Change maps of various disease severity stages were produced, so that the image information could find the indications and the $\mathrm{ShB}$ disease severity to scale it. Ground truth information gathering: The ground NDVI readings were taken with the help of the GreenSeeker handheld crop sensor. Various measurements were recorded in every field plot so that the accuracy of NDVI values increased which signified the severity of the ShB disease. In this work, 134 average NDVI readings were taken from the areas of 67 plots that were inoculated and un-inoculated with pathogens. After this ShB severity was rated on a scale of $0 \pm 9$ concerning disease symptoms. Data evaluation and drawing: For UAV image processing Pix4D mapper was used. ArcGIS 9.1 was used for mapping and geospatial study. For statistical evaluation PASW Statistic 18, software was used. RMSE and determination coefficient were used to find the accuracy of the correlation model. Françca et al. [21] developed a model for identifying D.Avenae fungi in black oat seeds using multispectral images. The study was performed using color and texture parameters from seeds incubated for 120 hours. Fig. 15 shows the raw image and relative grayscale and nCDA of black oat seeds. Results show the high performance of the model with an accuracy of 0.86 . This indicates that the multi-spectral imaging method was capable of recognizing D.Avenae fungi in black oat seeds. The accuracy achieved was 0.86 for the color and texture feature which was satisfactory. The accuracy achieved for black oat seeds inoculated for 24, 72, and 120 hours was $0.78,0.83$, and 1.00 respectively. This shows that multi-spectral imaging can be an adequate method that will assure that the black oat seeds do not contain any diseases or fungi.

2) Block Diagram to Detect D.Avenae Fungi in Black Oat Seeds: Data Acquisition: With the help of the VideometerLab4 instrument, 19 multispectral images of 200 non-infested seeds as well as 200 infested seeds were acquired (Fig. 16 c)). These seeds were infested with D. Avenae fungi for an inoculation interval of 24, 72, and 120 hours. 19 highresolution images were acquired at a time in 5 seconds. VideometerLab4 software version was used to analyze the data. The nCDA algorithm was employed to transform the images. This was performed so that there exists a maximum separation between the classes and minimum distance within a specific class. For every seed, the ROI was recognized and a mask was created to segment the seeds from the surrounding. The blob database was used to collect the seeds and from each seed, 36 variables were obtained. This also involved color components such as hue and saturation. The earlier acquired 19 multi-spectral images were then used to obtain the color features. Various color description models were employed to transform the multi-spectral data into color data. By calculating the individual spectral bands, the texture features were obtained. The 19 spectral wavelengths are in the range of $365 \mathrm{~nm}$ to $970 \mathrm{~nm}$. The mean reflectance of each seed lying within the range of the spectral bands was extracted with the help of the MultiColorMean feature. A trimmed mean of the transformed pixel values was calculated using RegionMSI (mean). Similarly, the percentage of the blob region that had a transformation value higher than the threshold was calculated using RegionMSIthresh. Data Evaluation: LDA method was used to develop two models that could differentiate between infested and non-infested seeds from the spectral information. The reflectance data obtained for each seed was used to create the first model. Similarly, color and texture features were used to create the second model. The data was split into two sets with $60 \%$ of each sample used for training the model and the remaining $40 \%$ of each sample for testing the model. The result of the model was evaluated using the kappa coefficient and accuracy. The VideometerLab4 and R software were used for statistical evaluation.

\section{RESULTS}

The results are summarised with the help of tables [Tables I to III] which concludes all the eight objectives of multispectral imaging of this manuscript in terms of their database size, number of color spectrums, accuracy, and other factors. Table I compares the various methods for varietal identification and quality analysis of fruits and vegetables. Shreshta et al. [8] has achieved the highest accuracy of $96 \%$ for the identification of varieties of 298 tomato seeds. Liu et al. classified 250 rice seeds samples based on their varieties. The accuracy achieved was $94 \%$. For the quality analysis of pomegranate fruits, Khodabakhshian et al. [10] considered 100 samples and achieved the highest accuracy of 97\% among the reported literature. Clemmensen et al. [9] had used the 747 number of samples for fruit quality analysis, which was the largest dataset used. A comparison of reported literature for leaf and tree species classification along with disease detection is shown in Table 2. The method proposed by Naeem et al. [17] for lemon balm leaf classification achieved a classification accuracy of $99.9 \%$ on a dataset of 600 images. Among the methods proposed for disease detection, Franca-Silva et al. [21] had achieved the highest accuracy of $100 \%$ for 400 samples by using 19 color spectrums. They had used the largest number of samples and the largest number of color spectrums. Table 3 compares reported literature in the areas of fruit/flower detection, internal fruit parameters, fruit ripeness, and fruit yield. For detection of foreign material in cabbage and green onion samples, Lohumi et al. [20] achieved an accuracy of greater than $95 \%$. For extracting quality parameters for 210 strawberry fruits, Liu et al. [1] has achieved $100 \%$ accuracy by using 19 different wavelengths. For classifying the fruit ripeness of 490 banana samples, Santoyo et al. [14] achieved the highest accuracy of $84 \%$. For predicting the fruit yield of sugar beet crops, Cao et al. [15] achieved the highest accuracy of $96.3 \%$ for 15 field plots. 


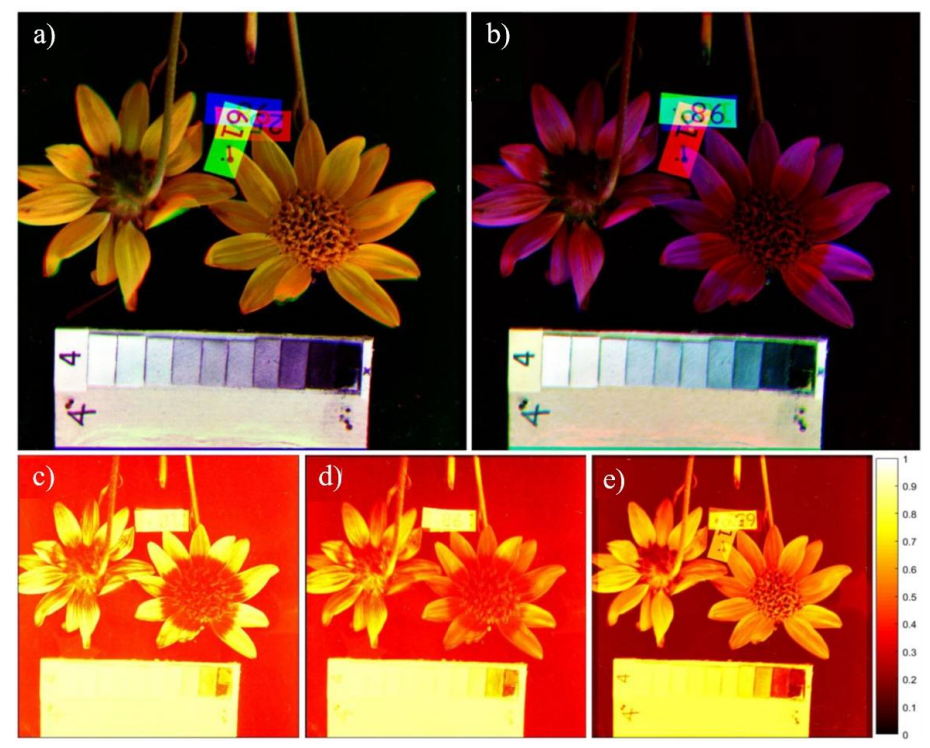

Fig. 17. A Series of Monochromatic Filters having Different Peak Transmissions are used to Capture Black-and-White Images. Further, a False-Color (FC) Image is Produced from these Photos to Correct the White Balance from the Images. These FC Images are Later Tinted with Black and White Images to Get a Proper Hue. (a) The 3 Layers i.e. RGB are Combined for Human Vision. (b) Transformation of Yellow, Blue and UV into Red, Green and Blue is Performed for the Bees. The Receptor Response is Calculated for the (c) Short (d) Medium (e) and Long-Wavelength Receptors which Explained the Electrical Signals that the Photoreceptors in Bees Generate in response to the Image [19].

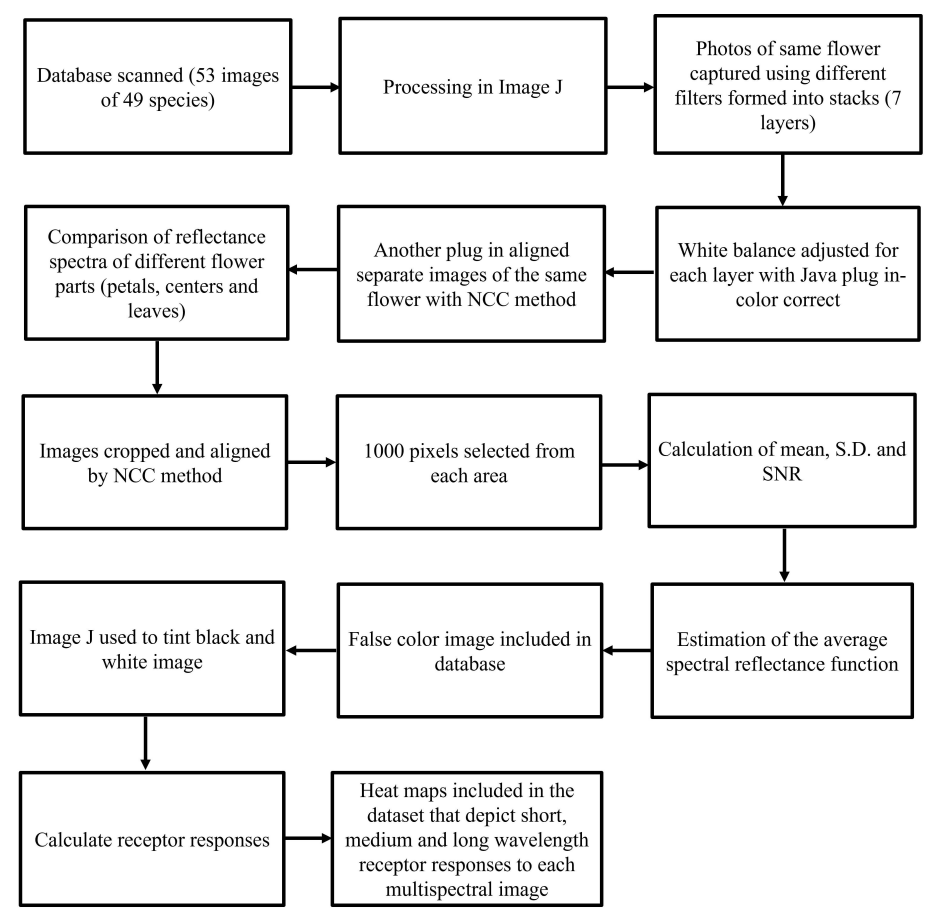

Fig. 18. Block Diagram of Fruit/Flower Detection using Long-Wavelength Sensitive Receptor for Bees. 


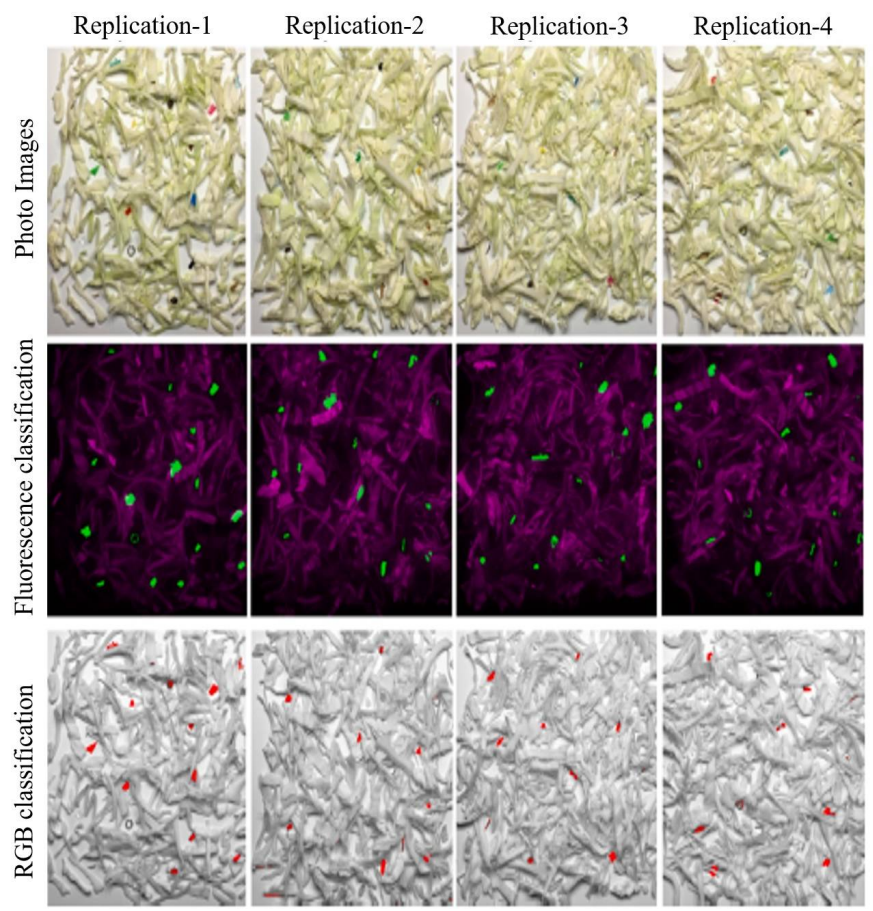

Fig. 19. Colour Images of Cabbage Samples Mixed with Foreign Materials (FM) are shown in the Top Row. Classification Images based on Fluorescence are shown in the Middle Row. Classification Images based on RGB are shown in the Bottom Row [20].

TABLE I. VARIETAL IDENTIFICATION AND FRUIT QUALITY

\begin{tabular}{|l|l|l|l|l|}
\hline $\begin{array}{l}\text { Sr. } \\
\text { No. }\end{array}$ & Method & Database size & $\begin{array}{l}\text { Number of colour } \\
\text { spectrums used }\end{array}$ & $\begin{array}{l}\text { Accuracy } \\
\text { (in \%) }\end{array}$ \\
\hline \multicolumn{5}{|c|}{ Varietal Identification } \\
\hline 1. & Liu et al. [13] & 250 seeds & 19 & 94 \\
\hline 2. & Shrestha et al. [8] & 298 seeds & 19 & 96 \\
\hline \multicolumn{5}{|c|}{ Fruit Quality } \\
\hline 1. & $\begin{array}{l}\text { Khodabakhshian } \\
\text { et al. [10] }\end{array}$ & 100 samples & 4 & 97 \\
\hline 2. & $\begin{array}{l}\text { Clemmensen } \\
\text { et al.[9] }\end{array}$ & 747 samples & 20 & - \\
\hline 3. & Lianou et al.[12] & 245 samples & 18 & 91.7 \\
\hline
\end{tabular}

\section{RESEARCH GAPS OF REPORTED LiterATURE}

For varietal identification of rice seeds, PLS-DA and PCABPNN models achieved low accuracies. PLS-DA and PCABPNN, even when tremendously used in chemometrics, does not give good results as in LS-SVM, at least for this case [1]. The spectroscopy method calculates the total amount of transmitted or reflected light from a certain part of a sample and does not contain wavelength-specific data [23]. This limitation was solved by using the hyperspectral imaging system which spatially receives the wavelength responses (pixel) of images of the fruit [10]. For each wavelength and each piece of vegetable, a t-test was conducted to check for significant differences on a $5 \%$ level in several percentiles of the light reflectance. The results show significant changes from days 2 to 4 in the reflectance spectrum for both the celeriac and carrots, significant changes continuing until day 14 were found [9]. A threshold value was set as TVC $\leq 2$ to assess the quality of the vanilla cream samples in the dairy industry to indicate a fresh sample. In earlier methods, finding the threshold limit was a costly, effort-intensive, and lengthy process. Thus, multispectral imaging provides a less expensive, fast, and automatic method that helps in the quick judgment process for quality managers [24]. To determine the quality features in the strawberry fruit, the limitation was that the NIR spectrometers can only identify a small part of the fruit not the entire fruit at certain times. The limitation of hyperspectral imaging is that problems are faced while processing the data that make it difficult for commercial real-time applications [25]. This problem is solved using multispectral imaging which enables a fast, nondestructive examination of the interior and exterior features of different types of vegetables and fruits [13]. The earlier methods that involve optical sensing, spectroscopy, and imaging are more complex in their algorithms or experimental setups, which are not useful in real-time applications. Other than this, earlier techniques never incorporated multispectral imaging in their work. So, this work focuses on multispectral imaging which helps in finding, recording, and quantifying the banana ripening process [14]. For observation of sugar beet growth indicators, WDRVI was used. Earlier studies used satellite platforms that are confined to both height and orbit and cannot manage the temporal, spatial, or spectral resolution essential for monitoring the growth [26]. The new UAV tech- 
TABLE II. Classification AND Disease Detection

\begin{tabular}{|l|l|l|l|l|}
\hline $\begin{array}{l}\text { Sr. } \\
\text { No. }\end{array}$ & Method & Database size & $\begin{array}{l}\text { Number of colour } \\
\text { spectrums used }\end{array}$ & Accuracy \\
\hline \multicolumn{5}{|c|}{ Classification } \\
\hline 1. & Naeem et al.[17] & 600 images & 5 & 99.9 \\
\hline 2. & $\begin{array}{l}\text { Abdollahnejad } \\
\text { et al.[18] }\end{array}$ & 297 images & 5 & 84.71 \\
\hline \multicolumn{5}{|c|}{ Disease Detection } \\
\hline 1. & Zhang et al.[22] & 67 field plots & 5 & 100 \\
\hline 2. & $\begin{array}{l}\text { Franca-Silva } \\
\text { et al.[21] }\end{array}$ & 400 samples & 19 & \\
\hline
\end{tabular}

TABle iII. Fruit/Flower Detection, Internal Fruit Parameter, Fruit RiPEness/Maturity and Fruit Yield.

\begin{tabular}{|l|l|l|l|l|}
\hline $\begin{array}{l}\text { Sr. } \\
\text { No. }\end{array}$ & Method & Database size & $\begin{array}{l}\text { No of colour } \\
\text { spectrums used }\end{array}$ & $\begin{array}{l}\text { Accuracy } \\
\text { (in \% })\end{array}$ \\
\hline \multicolumn{5}{|c|}{ Fruit/ flower detection } \\
\hline 1. & Vasas et al [19] & 53 samples & 7 & - \\
\hline 2. & Lohumi et al.[20] & - & 2 & $>95$ \\
\hline \multicolumn{5}{|c|}{ Internal fruit parameter } \\
\hline 1. & Liu et al.[1] & 210 fruits & 19 & 100 \\
\hline \multicolumn{7}{|c|}{ Fruit ripeness/ maturity } \\
\hline 1. & Santoyo et al.[14] & 490 images & 2 \\
\hline \multicolumn{7}{|c|}{ Fruit Yield } \\
\hline 1. & Cao et al. [15] & 15 plots & 5 \\
\hline
\end{tabular}

nology can manage low-cost, crop growth observation at high temporal, spatial, and spectral resolution. The RGB images acquired by the UAV evaluated the canopy estimated area of a tree and provided the best option. The LAI and leaf cluster biomass found from NDVI is used to monitor growth, but this is not possible for medium and high bio-masses. A WDRVI developed uses NVDI NIR band reflectance data, which causes lowering of the weights in such bands which belong to high and medium biomass cases [27]. This results in increased linearity and reduces saturation, which makes correct growth monitoring easier. An enhanced WDRVI is used to evaluate the early-phase growth of sugar beet [15]. In the classification of medicinal plant leaves, the feature selection (FS) process is the most crucial part of the ML-based classification. The goal of this research is to achieve better accuracy in less time. It is observed that without feature selection, the MLP classifier takes a lot of time (4.83 Seconds) to identify medicinal plants due to a large number of features. But when selected features are used, higher accuracy is obtained in less time. The PCA method is an unsupervised approach and does not provide good results on labeled data, as in this work the medicinal plant leaf varieties data set is labeled. This limitation in PCA is resolved by ML-based supervised feature selection techniques: chi-square feature evaluator (ranked search) method [28]. This method selects the best features from the huge features vector space (FVS). Compared to PCA, the current method can extract the sub-database with the best features for this large database [17]. Tree species classification studies are generally focused on rural and urban forests instead of managed forests. They are also limited to only spectral study and usually incorporate RGB sensors. Work on plant nurseries and low vegetation which has low tree volume and low levels of design complexity are useful, but forest conditions create problems for detection. SVM method was used with a combination of textural and spectral data. Later statistical methods for classifying tree species and identification of dead and unhealthy trees harmed by bark beetles are investigated [18]. The multispectral images allow us to model the visual response that the bees' photoreceptors give in response to the flower image. This, in turn, will help to differentiate the function of the different wavelength channels in color processing [19]. The earlier methods for detecting foreign objects in vegetables were metal detection, X-ray inspection, and color imaging techniques are not satisfactory. Also, the machine vision technology with the color camera is not useful for visibly opaque plastic FMs, and cannot detect the FMs that are similar in color to fresh-cut vegetables. The limitations of the earlier work were solved by using fluorescence imaging in this work [20]. The rice Sheath Blight (ShB) disease can be identified by using UAS, RGB, and multispectral imagery data. The color features computed using the multispectral images and the RGB images could hardly identify the variations in covering caused due to the disease [29]. Also, the information available is not enough to classify various levels of sheath blight, as the wavelength range is small and large bands related to the RGB camera are used [22]. For detection of D. Avenue fungi in black oat seeds, currently, the detection is done by examining the dry seed visually. This method is hard, slow, and requires experts. Advanced sensors combined with object recognition by computers that can automatically operate will result in a fast examination of seed health status by extraction of wavelength, texture features, and color [30]. Multispectral imaging combines optical spectroscopy and computer vision, which results in producing spatial and wavelength-specific data on different fungi species [21].

\section{FUTURE WORK}

\section{A. Varietal Identification}

For varietal identification of tomato, further studies will focus on the use of spectral data related to VIS-NIR obtained from multi-spectral imaging to discriminate the rice seeds of different varieties. This information will be related to practical organic aspects of tomato and other crops [8]. 


\section{B. Fruit Quality}

For quality evaluation of pomegranate fruit, future research will concentrate on the execution of the developed multispectral imaging by carrying out the real-time test [10]. For wok-fried vegetables of carrot and celeriac samples, the sensory quality of the wok-fried celeriac and carrots is assessed using the Quality Index Method (QIM). The scores of all features are then added that results in an overall sensory score. This is called Quality Index. QIM indicates scores of 0 for fresh products and larger scores in increasing order for spoiled products. This plan is observed by images and complete information of all factors. The sensory study requires that the samples are reheated before assessment, as otherwise, it is not possible to assess taste and smell [9]. For vanilla cream, microbiological quality classification the Unsupervised Online Feature Selection (UOS) algorithm could manage a classification error that was quite low in the test database. The threshold limit which was set for the "fresh" samples of vanilla cream, was a crucial factor for product delivery to the market by the manufacturer. This example gives a good outlook for the application of multi-spectral imaging in combination with the UOS algorithm in the quality management of the dairy industry [12].

1) Internal Fruit Parameter: To find quality features and ripeness phase in strawberry fruit, PLS, SVM, and BPNN models were employed. All the models' used visible areas of the spectrum. But, if the NIR spectra are also incorporated then the results could be marginally improved. The multi-spectral imaging system can reduce the image retrieval as well as operating time in contrast to the hyper-spectral imaging system that allows online automated quality observation systems [13].

2) Fruit Ripeness/Maturity: For the ripening process in bananas, future work can be online applications to measure maturity level in other fruits [14].

3) Fruit Yield: For observation of sugar beet growth indicators, the future work can be for growth tracking of potato, radish, and other underground crops to a large extent for reaping benefits [15].

\section{Classification}

For the classification of medicinal plant leaves, the reported study was limited to six medicinal plant leaves while there are millions of types of medicinal plant/herbs in the world. This is a pixel-based method and in the future, an objectbased method can be used. In the future, this proposed method can be used on other medicinal plant leaves. Further, the results can be improved using hyper-spectral and 3D digital image data sets [17]. For tree species classification and health status assessment, identifying physical stress in earlier stages or finding small wavelength changes can be done with high reliability and accuracy by using hyper-spectral sensors [31]. This was not possible in multi-spectral sensors as it does not operate in that wavelength range. Future work can be on the application of thermal sensors which will enhance the classification and health status of different tree species. The combination of multi-spectral sensors and UAS can decrease the operating costs of tracking forest areas in the small to medium range [18].
1) Fruit/Flower Detection: For multi-spectral images of flowers, receptors with long wavelengths for edge detection in bees were used. The results provide acceptable information for selecting the long-wavelength channel over the shorterwavelength channels for clarifying a visual scene. This can initiate the ways environment and prior knowledge can build more resulting neural processes which can be the future work [19]. For real-time detection of foreign material mixed with fresh-cut vegetables, two types of fresh-cut vegetables were tested. For future work, this method can be experimented on different vegetables by picking the best fluorescence wavelengths(band) and using a suitable algorithm for processing images of fresh-cut vegetables [20].

\section{Disease Detection}

For detection of rice sheath blight, using a UAV can help in the cultivation of rice cultivars with resistance to Sheath Blight disease. For future work, a new UAV system developed can be used for site-specific accurate fungicide application technique to control ShB disease in rice [22]. For the detection of D. Avenae in black oat seeds, multi-spectral imaging can be used in the future for separating seed-carrying fungi, affected by other elements, but are firmly related to physical and chemical differences caused by fungi [21].

\section{CONCLUSION}

In this manuscript, we have multi-spectral imaging which is the emerging technology for the grading of fruits and vegetables. Quality assessment of vegetables and fruits is the need of the hour for the food industry. To meet consumer demand and profit, the technologies developed should provide more concise, fast, and accurate results. Some of these technologies have their merits and demerits. We have reviewed different ways in which multi-spectral images could be used for fruit and vegetable assessment like varietal identification which includes identification of rice seeds and tomato, fruit quality which includes wok-fried vegetables(carrots and celeriac), quality of vanilla cream, etc. Such images can also be used classification of medicinal plant leaves and tree species, rice sheath blight disease detection, identifying pathogens in black oat seeds, etc. Multi-spectral imaging also enables the detection of foreign material in cabbage, to find how the honey bees and bumblebees respond to images of flowers for long, short, and medium wavelength receptors. Internal fruit parameters like firmness and TSS in strawberries can also be extracted. Other applications of multi-spectral imaging include the estimation of fruit ripeness and fruit yield. The data obtained by multispectral imaging can enable the evaluation of the classification of fruits and vegetables. The study of multi-spectral imaging for fruit and vegetable will be beneficial to disease prevention, irrigation, and yield improvement. Furthermore, multi-spectral information can be considered the basis for evaluating various characteristics and parameters of fruit, vegetable, and tree species. It would also promote research on fruit, vegetable, and trees species.

\section{DECLARATIONS}

\section{A. Funding Information}

No funding was involved in the present work. 


\section{B. Conflicts of Interest}

Authors S. Gaikwad and S. Tidke declare that there has been no conflict of interest.

\section{Code Availability}

Not applicable.

\section{Authors' Contributions}

Conceptualization was done by Shilpa Gaikwad (SG) and Sonali Tidke (ST). All the literature reading and data gathering were performed by SG. The formal analysis was performed by SG. Manuscript writing and original draft preparation was done by SG. Review and editing was done by ST. Visualization work was carried out by SG and ST.

\section{E. Ethics Approval}

All authors consciously assure that the manuscript fulfills the following statements: 1) This material is the authors' own original work, which has not been previously published elsewhere. 2) The paper is not currently being considered for publication elsewhere. 3) The paper reflects the authors' own research and analysis in a truthful and complete manner. 4) The paper properly credits the meaningful contributions of coauthors and co-researchers. 5) The results are appropriately placed in the context of prior and existing research.

\section{F. Involvement of Human Participant and Animals}

All the necessary permissions were obtained from the Institute Ethical Committee and concerned authorities to run our algorithms on patient data.

\section{G. Information about Informed Consent}

Informed consent was obtained from participants whose data was used to do analysis.

\section{H. Consent for Publication}

Authors have taken all the necessary consents for publication wherever required.

\section{REFERENCES}

[1] W. Liu, C. Liu, F. Ma, X. Lu, J. Yang, and L. Zheng, "Online variety discrimination of rice seeds using multispectral imaging and chemometric methods," Journal of Applied Spectroscopy, vol. 82, no. 6, pp. 993-999, 2016.

[2] T. Bourlai and B. Cukic, "Multi-spectral face recognition: Identification of people in difficult environments," in 2012 IEEE International Conference on Intelligence and Security Informatics. IEEE, 2012, pp. 196-201.

[3] M. Moroni, A. Mei, A. Leonardi, E. Lupo, and F. L. Marca, "Pet and pvc separation with hyperspectral imagery," Sensors, vol. 15, no. 1, pp. 2205-2227, 2015.

[4] H.-W. Chen, J.-H. Lee, B.-Y. Lin, S. Chen, and S.-T. Wu, "Liquid crystal display and organic light-emitting diode display: present status and future perspectives," Light: Science \& Applications, vol. 7, no. 3, pp. $17168-17168,2018$

[5] J. D. Vincent, S. Hodges, J. Vampola, M. Stegall, and G. Pierce, Fundamentals of Infrared and Visible Detector Operation and Testing. John Wiley and Sons, 2015.

[6] I. Makki, R. Younes, C. Francis, T. Bianchi, and M. Zucchetti, "A survey of landmine detection using hyperspectral imaging," ISPRS Journal of Photogrammetry and Remote Sensing. vol. 124. pp. 40-53, 2017.
[7] B. Zhang, W. Huang, J. Li, C. Zhao, S. Fan, J. Wu, and C. Liu, "Principles, developments and applications of computer vision for external quality inspection of fruits and vegetables: A review," Food Research International, vol. 62, pp. 326-343, 2014.

[8] S. Shrestha, L. C. Deleuran, M. H. Olesen, and R. Gislum, "Use of multispectral imaging in varietal identification of tomato," Sensors, vol. 15, no. 2, pp. 4496-4512, 2015.

[9] L. H. Clemmensen, B. S. Dissing, G. Hyldig, and H. Løje, "Multispectral imaging of wok-fried vegetables," Journal of Imaging Science and Technology, vol. 56, no. 2, pp. 20 404-1, 2012.

[10] R. Khodabakhshian, B. Emadi, M. Khojastehpour, M. R. Golzarian, and A. Sazgarnia, "Development of a multispectral imaging system for online quality assessment of pomegranate fruit," International Journal of Food Properties, vol. 20, no. 1, pp. 107-118, 2017.

[11] A. Bhargava and A. Bansal, "Grading of variety of bi and mono-colored applest," in Soft Computing and Signal Processing. Springer, 2022, pp. 375-382.

[12] A. Lianou, A. Mencattini, A. Catini, C. Di Natale, G.-J. E. Nychas, E. Martinelli, and E. Z. Panagou, "Online feature selection for robust classification of the microbiological quality of traditional vanilla cream by means of multispectral imaging," Sensors, vol. 19, no. 19, p. 4071, 2019.

[13] C. Liu, W. Liu, X. Lu, F. Ma, W. Chen, J. Yang, and L. Zheng, "Application of multispectral imaging to determine quality attributes and ripeness stage in strawberry fruit," PloS one, vol. 9, no. 2, p. e87818, 2014.

[14] M. Santoyo-Mora, A. Sancen-Plaza, A. Espinosa-Calderon, A. I. Barranco-Gutierrez, and J. Prado-Olivarez, "Nondestructive quantification of the ripening process in banana (musa aab simmonds) using multispectral imaging," Journal of Sensors, vol. 2019, 2019.

[15] Y. Cao, G. L. Li, Y. K. Luo, Q. Pan, and S. Y. Zhang, "Monitoring of sugar beet growth indicators using wide-dynamic-range vegetation index (wdrvi) derived from uav multispectral images," Computers and Electronics in Agriculture, vol. 171, p. 105331, 2020.

[16] L. Wittstruck, I. Kühling, D. Trautz, M. Kohlbrecher, and T. Jarmer, "Uav-based rgb imagery for hokkaido pumpkin (cucurbita max.) detection and yield estimation," Sensors, vol. 21, no. 1, p. 118, 2021.

[17] S. Naeem, A. Ali, C. Chesneau, M. H. Tahir, F. Jamal, R. A. K. Sherwani, and M. Ul Hassan, "The classification of medicinal plant leaves based on multispectral and texture feature using machine learning approach," Agronomy, vol. 11, no. 2, p. 263, 2021.

[18] A. Abdollahnejad and D. Panagiotidis, "Tree species classification and health status assessment for a mixed broadleaf-conifer forest with uas multispectral imaging," Remote Sensing, vol. 12, no. 22, p. 3722, 2020.

[19] V. Vasas, D. Hanley, P. G. Kevan, and L. Chittka, "Multispectral images of flowers reveal the adaptive significance of using long-wavelengthsensitive receptors for edge detection in bees," Journal of Comparative Physiology A, vol. 203, no. 4, pp. 301-311, 2017.

[20] S. Lohumi, B.-K. Cho, and S. Hong, "Lctf-based multispectral fluorescence imaging: System development and potential for real-time foreign object detection in fresh-cut vegetable processing," Computers and Electronics in Agriculture, vol. 180, p. 105912, 2021.

[21] F. França-Silva, C. H. Q. Rego, F. G. Gomes-Junior, M. H. D. d. Moraes, A. D. d. Medeiros, and C. B. d. Silva, "Detection of drechslera avenae (eidam) sharif [helminthosporium avenae (eidam)] in black oat seeds (avena strigosa schreb) using multispectral imaging," Sensors, vol. 20, no. 12 , p. $3343,2020$.

[22] D. Zhang, X. Zhou, J. Zhang, Y. Lan, C. Xu, and D. Liang, "Detection of rice sheath blight using an unmanned aerial system with highresolution color and multispectral imaging," PloS one, vol. 13, no. 5, p. e0187470, 2018.

[23] A. Ghita, P. Matousek, and N. Stone, "High sensitivity non-invasive detection of calcifications deep inside biological tissue using transmission raman spectroscopy," Journal of biophotonics, vol. 11, no. 1, p. e201600260, 2018.

[24] G. ElMasry, N. Mandour, S. Al-Rejaie, E. Belin, and D. Rousseau, "Recent applications of multispectral imaging in seed phenotyping and quality monitoring-an overview," Sensors, vol. 19, no. 5, p. 1090, 2019. 
[25] T. Adão, J. Hruška, L. Pádua, J. Bessa, E. Peres, R. Morais, and J. J. Sousa, "Hyperspectral imaging: A review on uav-based sensors, data processing and applications for agriculture and forestry," Remote Sensing, vol. 9, no. 11, p. 1110, 2017.

[26] W. H. Maes and K. Steppe, "Perspectives for remote sensing with unmanned aerial vehicles in precision agriculture," Trends in plant science, vol. 24, no. 2, pp. 152-164, 2019.

[27] L. Wang, Q. Chang, F. Li, L. Yan, Y. Huang, Q. Wang, and L. Luo, "Effects of growth stage development on paddy rice leaf area index prediction models," Remote Sensing, vol. 11, no. 3, p. 361, 2019.

[28] S. Picek, A. Heuser, A. Jovic, and L. Batina, "A systematic evaluation of profiling through focused feature selection," IEEE Transactions on Very Large Scale Integration (VLSI) Systems, vol. 27, no. 12, pp. 2802
$2815,2019$.

[29] S. Thomas, M. T. Kuska, D. Bohnenkamp, A. Brugger, E. Alisaac, M. Wahabzada, J. Behmann, and A.-K. Mahlein, "Benefits of hyperspectral imaging for plant disease detection and plant protection: a technical perspective," Journal of Plant Diseases and Protection, vol. 125, no. 1, pp. 5-20, 2018.

[30] S. Cubero, N. Aleixos, E. Moltó, J. Gómez-Sanchis, and J. Blasco, "Advances in machine vision applications for automatic inspection and quality evaluation of fruits and vegetables," Food and bioprocess technology, vol. 4, no. 4, pp. 487-504, 2011.

[31] A. Lowe, N. Harrison, and A. P. French, "Hyperspectral image analysis techniques for the detection and classification of the early onset of plant disease and stress," Plant methods, vol. 13, no. 1, pp. 1-12, 2017. 